\title{
CLINICAL CASE OF FILLING MATERIAL FORCED IN THE MANDIBULAR CANAL (CLINICAL CASE AND TREATMENT)
}

\author{
Georgiev T., Edrev S. \\ Department of Oral and Maxillofacial Surgery, Faculty of Dental Medicine, \\ Medical University - Varna, Bulgaria \\ Department of Oral Surgery, Dental Centre №1, Varna, Bulgaria
}

\begin{abstract}
:
Until not so distant past, in our country it was considered that endodontic treatment of the teeth in all forms of chronic periodontitis should be completed by forcing the filling material outside the borders of the apex of the treated tooth. Nowadays, this approach is seriously reconsidered.
\end{abstract}

Key words: complications in endodontic treatment, paresthesia, neuritis of n.alveolaris inferior, surgical treatment

Nowadays one of the most frequently met mistake in endodontic treatment is considered to be the forcing of the filling material outside the borders of the apex of the tooth root.

This complication occurs as a result of:

- incorrect determining of the length of the root canal as a result of which the endodontic tools perforate the apex and mechanical treatment is carried out by passing the limits of the canal into the periapical tissues;

- using poor endodontic equipment;

- the use of mechanical root fillers at a high speed always leads to forcing the filling material outside the tooth apex etc.

As regards the indications for surgical treatment of this complication, they can be divided in absolute and relative.

They are relative: when the root-filling material which is outside the limits of the tooth apex does not cause pain, there is no aggravation of the process, in case of small size of material in the periapical tissues. In these cases, it is recommended the patient to be observed over a period of 3-6-12 months.

The absolute indications for surgical removal of the root-filling material are:

- inserted root-filling material in the lower jaw canal;

- presence of considerable quantities of root-filling material in the upper jaw sinus;

- radicular cyst with forced root-filling material in the cavity ];
- expressed pain reaction for a period of 14 days;

- presence of foreign body in the tissues which maintains inflammation etc.

In all of the above cases, surgical treatment of the problem has to be undertaken.

\section{CLINICAL CASE}

A 20-year old girl was admitted for treatment in the surgical department due to sharp pain in the region of all teeth on the right lower jaw, the pain originated as a result of endodontic treatment of 45 tooth. Four days had passed from the moment of filling the tooth until the patient was admitted for treatment. During this period the pain did not stop although painkillers were regularly taken - Analgin, Veronal, Aulin - they did not help. In the last two days the personal doctor injected her with Tramal at every 6 hours the only therapeutic agent which reduced the pain. The patient was made an X-ray which clearly shows the reason for these complaints - forced root-filling material which goes outside the limits of the root canal of 45 tooth and reached the cyst around the root and the mandibular canal (the filling in the canal reaches about $1 \mathrm{~cm}$ ) Pict. 1.

Established diagnosis: foreign body in the area of the mandibular canal on the right.

Under venous anesthesia with Dormicum and Fentanyl, as well as local conduction anesthesia with Mepivastesin 3\%, mucoperiosteal flap was mobilized on the vestibular side of the alveolar ridge of the lower jaw in the region of 44-47 teeth, the flap was carefully laid back in the region of 45 tooth until visualization of foramen mantale, after that the exterior wall of the mandibular canal was removed (Pict. 2).

Cystectomy was made of 45 tooth and extraction of 46 tooth due to the fact that more than $1 / 3$ of the tooth apex was engaged in the process, the root-filling material was removed from the canal with the help of blunt surgical tools, and the wound was periodically washed with antiseptic solutions. Silk 3-0 sutures were made and a drainage was inserted in the wound which later was removed on the $2^{\text {nd }}$ day. 
In the post-surgical period we noted moderate oedema of the tissues in the region of the surgery, the temperature was normal, the mucosa was of normal pink colour, another segment X-ray was made (Pict. 3).

After the surgery the patient was preventively prescribed antibiotics and analgesics.

There were no complaints after the surgery as regards pain, the patient reported numbness of the lower lip which continued for about a week altogether, and gradually, day after day its sensitivity was restored.

\section{DISCUSSION:}

It has to be noted that the success of the surgical treatment and respectively the course of the post-surgical period are dependent on the time of locating the material and its effect on the surrounding osseous and nervous tissue. The sooner it is removed from the mandibular canal, the smoother is the post-surgical period and sensitivity is faster restored.
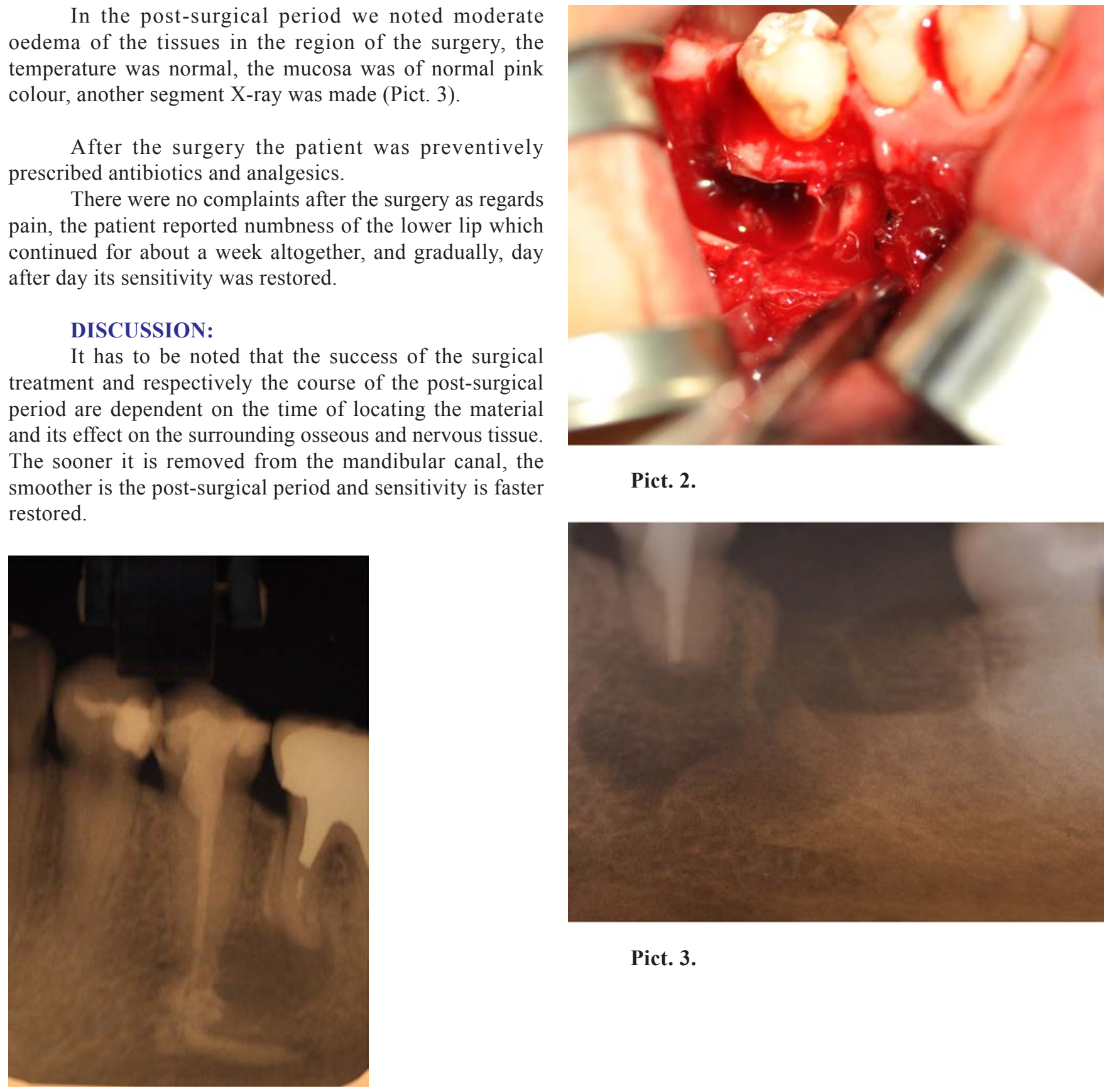

Pict. 2.

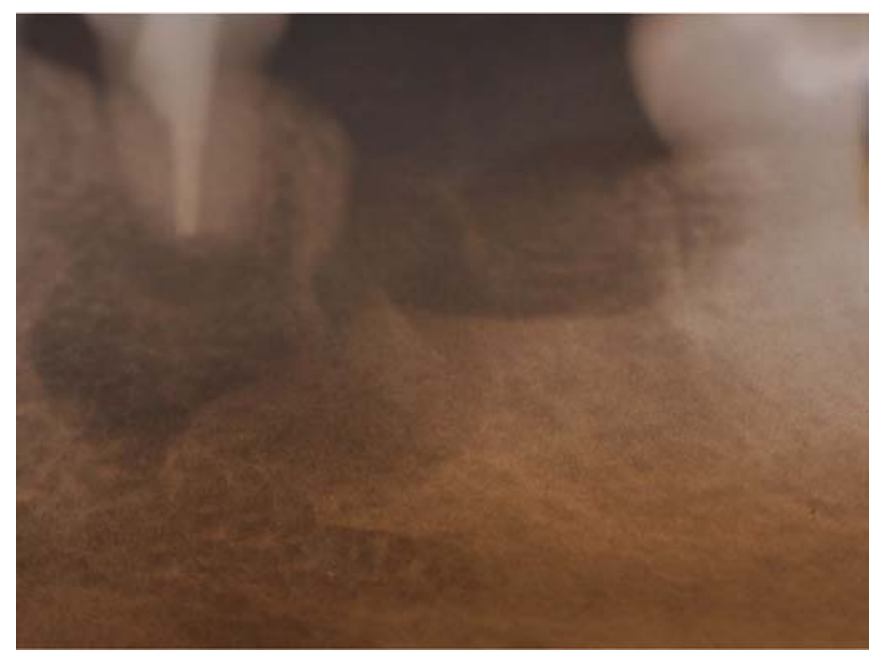

Pict. 3.

Pict. 1 


\section{REFERENCES:}

1. Meyer RA, Bagheri SC. A bioabsorbable collagen nerve cuff (NeuraGen) for repair of lingual and inferior alveolar nerve injuries: a case series. J Oral Maxillofac Surg. 2009 Nov; 67(11):2550-1

2. Bagheri SC, Meyer RA, Khan HA, Steed MB. Microsurgical repair of peripheral trigeminal nerve injuries from maxillofacial trauma. J Oral Maxillofac Surg. 2009 Sep;67(9):1791-9.
3. Ch. Babush. Transpositioning and retranspositioning the inferior alveolar and mental nerves in conjunction with endosteal implant reconstruction. J. Periodontology 2000, vol.17,1998, pp.183190

4. Solar P., Glm C.,Frey G Matejka M. A classification of the intraosseous paths of the mental nerve. $J$ of Oral and Maxillofacial implants 1994:9:339:344.

5. Wismeijer D., Van Wass MAJ,
Vermeeren JIJF. Kalk W. Patients perception of sensory disturbances of the mental nerve before and after implant surgery.A prospective study of 110 patients. Br.J. Oral and Maxillofacial surgery 1997:35:254:259

6. Tolstunov L, Pogrel MA. Delayed paresthesia of inferior alveolar nerve after extraction of mandibular third molar: case report and possible etiology. J Oral Maxillofac Surg. 2009 Aug;67(8):1764-6.
Correspondence address:

Dr Tihomir Georgiev

18 Hristo Popovich St., Varna 9000, Bulgaria

e-mail: tgeorgeivphd@yahoo.com 